\title{
Reprodutibilidade da Medida Ambulatorial da Pressão Arterial em Pacientes Hipertensos com Diabete Melito Tipo 2
}

\author{
Reproducibility of Ambulatory Blood Pressure Monitoring in Hypertensive Patients with Type 2 Diabetes Mellitus
}

João Soares Felício, Juliana Torres Pacheco, Sandra Roberta Ferreira, Frida Plavnik, Oswaldo Kohlmann, Artur Beltrame Ribeiro, Maria Tereza Zanella

Divisão de Nefrologia e Endocrinologia da UNIFESP - São Paulo, SP

\section{Resumo}

Objetivo: Avaliar a reprodutibilidade e o efeito placebo sobre a monitorização ambulatorial da pressão arterial (MAPA) (Spacelabs-90207).

Métodos: Mensurou-se a PA no consultório e por meio de duas MAPA, realizadas em um intervalo de 1 a 10 meses (média de 4,9 meses), de 26 pacientes com diabetes tipo 2 e hipertensão. Onze pacientes (G1) realizaram as duas MAPA sem medicação anti-hipertensiva por 15 dias, enquanto o G2 $(\mathrm{N}=15)$ fez a segunda MAPA em uso de placebo pelo mesmo período.

Resultados: Ao avaliarmos os coeficientes de variação (CV) da PA sistólica na vigília (PASV), PA diastólica na vigília (PADV), PA sistólica nas 24h (PAS24h) e PA diastólica nas 24h (PAD24h), encontramos valores de 4,6\%, 3,9\%, 5,0\% e 4,0\% no G1 e 4,3\%, 5,1\%, 3,7\% e 5,1\% no G2, respectivamente. Quanto ao CV da PA sistólica e diastólica durante o sono (PASS e PADS), encontramos 7,7\% e 8,2\% para G1, e 5,6\% e 6,3\% para G2, respectivamente. O CV da freqüência cardíaca na vigília e durante o sono foram: G1 = 5,9\% e 9,0\%, G2 = 6,9\% e 5,8\%, respectivamente. Analisando o total dos pacientes, todas as variáveis mostraram fortes correlações entre a primeira e a segunda MAPA (PASV, $r=0,76$; $\mathrm{P}<0,001 ; \mathrm{PADV}, \mathrm{r}=0,65 ; \mathrm{p}<0,001 ; \mathrm{PAS24h}, \mathrm{r}=0,77 ; \mathrm{p}<0,001 ; \mathrm{PAD24h}, \mathrm{r}=0,70 ; \mathrm{p}<0,001 ; \mathrm{PASS}, \mathrm{r}=0,62 ; \mathrm{p}<0,001$; PADS, $r=0,52 ; p<0,01)$. Ocorreram também correlações entre a PA sistólica e a diastólica de consultório e a PAS24h e PAD24h $(r=0,65 ; p<0,001 ; r=0,57 ; p<0,01)$.

Conclusão: A média dos níveis pressóricos avaliados pela MAPA apresentou boa reprodutibilidade e esses não foram afetados pelo efeito placebo.

Palavras-chave: Diabete melito tipo 2, hipertensão arterial, monitorização ambulatorial da pressão arterial.

\begin{abstract}
Summary
Objective: To evaluate the reproducibility of ambulatory blood pressure monitoring (ABPM) (SpaceLabs-90207) and placebo effect on ABPM.

Methods: Blood pressure was measured in the office and over two ABPM periods with an interval from one to ten months (mean 4.9 months), in 26 patients with type 2 diabetes mellitus and hypertension. Eleven patients (G1) had two ABPMs without taking antihypertensive drugs for 15 days, whereas $G 2(N=15)$ had the second ABPM after administration of a placebo for 15 days.

Results: In the evaluation of the coefficient of variation (CV) of diurnal (awake) systolic BP (DSBP), of diurnal (awake) diastolic BP (DDBP), of 24-hour systolic BP (24hSBP) and of 24-hour diastolic BP (24hDBP), the values found were $4.6 \%, 3.9 \%, 5.0 \%, 4.0 \%$ for G1 and $4.3 \%, 5.1 \%$, $3.7 \%, 5.1 \%$ for G2 respectively. We also determined the CV of nocturnal (sleep) systolic and diastolic BP (NSBP and NDBP) for G1 (7.7\%; $8.2 \%)$ and G2 (5.6\%; 6.3\%). Heart rate CV during alertness and sleep were: $G 1=5.9 \%$ and $9.0 \% ; G 2=6.9 \%$ and $5.8 \%$ respectively. When the total number of 'patients was analyzed, all variables showed a strong correlation between the first and second ABPM measurements (DSBP, $r=0.76$; $P<0.001 ; D D B P, r=0.65 ; p<0.001 ; 24 h S B P, r=0.77 ; p<0.001 ; 24 h D B P, r=0.70 ; p<0.001 ; N S B P, r=0.62 ; p<0.001 ; N D B P, r=$ $0.52 ; p<0.01)$. Office systolic and diastolic BP and 24hSBP and 24hDBP also showed correlation $(r=0.65 ; p<0.001 ; r=0.57 ; p<0.01)$.

Conclusion: Mean of pressure levels measured by ABPM presented good reproducibility and were not affected by placebo.
\end{abstract} Key words: Diabetes mellitus, type 2; hypertension; blood pressure monitoring, ambulatory.

Correspondência: João Soares Felício •

Rua Ferreira Cantão, 454 - S/308 - 66015-280 - Belém, PA

E-mail: felicio.bel@terra.com.br

Artigo recebido em 7/01/06; revisado recebido em 28/05/06; aceito em 22/06/06. 


\section{Artigo Original}

\section{Introdução}

A hipertensão arterial ocorre com uma freqüência duas vezes maior em pacientes com diabete melito (DM) quando comparados a indivíduos normais ${ }^{1,2}$. As complicações macro e microvasculares do DM também são aceleradas pela presença de hipertensão $o^{3,4}$. Portanto, tem sido recomendado que pacientes hipertensos e diabéticos devam ser diagnosticados e tratados precocemente ${ }^{5}$. Entretanto, como a pressão arterial (PA) é um parâmetro muito variável, a técnica padrão para estabelecer o diagnóstico de hipertensão permanece controversa ${ }^{6}$.

Durante os últimos anos, a monitorização ambulatorial da pressão arterial (MAPA) foi introduzida no estudo da hipertensão e tem se tornado um instrumento importante para a tomada de decisões clínicas ${ }^{7}$. Vários dados ${ }^{8-10}$ têm sugerido que a média da PA de 24 horas apresenta melhor correlação com as lesões em órgão-alvo que as medidas isoladas da PA no consultório. Também tem sido demonstrado que a MAPA apresenta maior reprodutibilidade quando comparada à PA de consultório, tanto em hipertensos ${ }^{11,12}$ quanto em diabéticos hipertensos ${ }^{13}$. Atualmente, a MAPA pode ser particularmente útil em diabéticos hipertensos para a detecção de disfunção autonômica e elevação da carga pressórica noturna ${ }^{14}$. Uma ausência ou diminuição da queda de PA durante o sono (nondipping) tem sido associada a um maior risco de complicações cardiovasculares $^{15,16}$

Adicionalmente, grandes diferenças de um dia para o outro têm sido reportadas em estudos de reprodutibilidade para a média da PA de 24 horas $^{17}$ e para a queda de PA durante o sono ${ }^{18}$ aferidas pela MAPA, especialmente em decorrência da diversidade de equipamentos existentes no mercado, muitos dos quais ainda não suficientemente testados. O estabelecimento do coeficiente de variação dos equipamentos de MAPA tornará seus resultados mais confiáveis.

Este estudo, portanto, foi desenhado para avaliar a reprodutibilidade e o efeito placebo na MAPA para determinar o seu grau de confiabilidade na mensuração dos níveis pressóricos em pacientes com DM tipo 2 e hipertensão.

\section{Métodos}

Pacientes - Vinte e seis pacientes com DM tipo 2 e hipertensão arterial, atendidos em nossa Clínica de Hipertensão durante um ano, foram avaliados e submetidos a MAPA em duas oportunidades, em um intervalo que variou de 1 a 10 meses (média de 4,9 meses). Inicialmente, todos os pacientes realizaram a primeira MAPA sem medicação antihipertensiva por 15 dias. Então, os pacientes foram divididos em dois grupos: o Grupo 1 (G1) $(\mathrm{N}=11)$ repetiu o mesmo procedimento para a segunda MAPA, enquanto o Grupo 2 (G2) $(\mathrm{N}=15)$ fez a segunda MAPA em uso de placebo durante os 15 dias. A pressão arterial de consultório foi também determinada para cada paciente no dia da colocação do aparelho da MAPA. Como critérios de inclusão, todos os pacientes deviam apresentar idade entre 30 e 75 anos, índice de massa corpórea (IMC) < ou igual a $40 \mathrm{~kg} / \mathrm{m}^{2}$, DM tipo 2, tratados somente com dieta ou hipoglicemiantes orais (sem uso de insulina), hipertensão arterial, níveis normais de creatinina sérica e proteinúria de 24 horas $(<150 \mathrm{mg} / 24 \mathrm{~h})$ e ausência de história prévia de infarto do miocárdio, angina e acidente vascular cerebral. Os indivíduos que não se enquadrassem nos critérios citados seriam excluídos do estudo. Os pacientes foram posteriormente classificados segundo estágio de hipertensão ${ }^{19}$ baseado na média de três medidas da PA de consultório aferida em posição sentada, com intervalo de um minuto entre as medidas, as quais foram realizadas no dia da primeira MAPA.

Hipertensão arterial foi definida como PA sistólica e diastólica aferida no consultório $\geq 140$ / 90 mmHg em medidas repetidas $^{20}$. O diagnóstico de DM foi baseado em critérios padrão ${ }^{21}$. Pacientes com DM tipo 2 foram identificados como aqueles com manifestação da doença após os 30 anos e sem necessidade de uso de insulina desde o diagnóstico. Todos os pacientes foram informados previamente de todos os procedimentos a que seriam submetidos e concordaram em participar do estudo. O protocolo de avaliação foi aprovado pelo comitê de ética da Universidade Federal de São Paulo.

Monitorização ambulatorial da pressão arterial - A MAPA foi realizada pelo método oscilométrico com o monitor portátil SpaceLabs - 90207 (Spacelabs, Inc. Redmond, WA-USA). Os pacientes realizaram a MAPA após a suspensão da medicação anti-hipertensiva por 15 dias ou após uso de placebo pelo mesmo período. O monitor foi instalado no período da manhã e retirado após 24 horas. O indivíduo era orientado para manter as suas atividades habituais e apresentar um relatório no qual deveria constar o horário de cada atividade realizada. O dispositivo foi programado para realizar quatro medidas durante cada hora, sendo estabelecida a média pressórica sistólica e diastólica durante cada hora, no período de vigília, durante o sono e nas 24 horas.

O período de vigília incluiu atividades de 8 horas a 20 horas, enquanto o período de 20 horas a 8 horas foi considerado como o período durante o sono. Medidas de PA sistólica maiores que $260 \mathrm{mmHg}$ e menores que $70 \mathrm{mmHg}$ e da PA diastólica superiores a $150 \mathrm{mmHg}$ e inferiores a 40 $\mathrm{mmHg}$ eram automaticamente excluídas da análise. O limite para detecção da freqüência cardíaca foi entre 200 e 20 bpm. O exame foi aceito se pelo menos 75\% das medidas nas 24 horas fossem executadas com sucesso. Adicionalmente, foi realizado o cálculo do descenso da PA durante o sono (DS) por meio da seguinte fórmula: DS sistólico (\%) = (PA sistólica na vigília - PA sistólica durante o sono) x 100 / PA sistólica na vigília; o mesmo cálculo foi utilizado para determinar o DS diastólico. Consideraram-se normais indivíduos que apresentaram descenso da PA durante o sono em relação a vigília maior ou igual a $10 \%$ (dippers), e os indivíduos que apresentaram queda inferior a esses níveis foram chamados de "non-dippers".

Análise estatística - Todos os dados foram apresentados na forma de média \pm desvio padrão ou mediana com intervalo de variação. Para análise de correlação, os coeficientes de correlação de "Pearson" ou de "Spearman" foram calculados entre variáveis, considerando-se sua distribuição normal ou não na população. Foi calculado ainda o coeficiente de variação $(\mathrm{CV})$ para todas as médias pressóricas e para a freqüência cardíaca da primeira e da segunda MAPA. O CV consistiu no desvio padrão da média da diferença dividido pela média absoluta das duas amostras multiplicada por 100 . 
Um $\mathrm{p}<0,05$ foi considerado significante. Todas as análises foram realizadas utilizando-se o programa Sigmastat (19921994 - Jandel Scientific Corporation- USA).

\section{Resultados}

A idade e o IMC dos dois grupos foram: $\mathrm{G} 1=59 \pm 8$ anos e $31 \pm 5 \mathrm{~kg} / \mathrm{m}^{2}$ e G2=56 \pm 8 anos e $27 \pm 4 \mathrm{~kg} / \mathrm{m}^{2}$, respectivamente. As medidas pressóricas avaliadas pela MAPA e divididas por grupo encontram-se na tabela 1. Não houve diferença estatisticamente significante em todas as medidas avaliadas pela MAPA entre os dois grupos e dentro de cada grupo quando comparadas a primeira e a segunda MAPA (tab. 1). Com relação ao estágio de hipertensão dos pacientes do G1, 3 pacientes (27\%) encontravam-se no estágio I, 6 (55\%) no estágio II, e 2 (18\%) no estágio III; enquanto os pacientes do G2 encontravam-se 2 (13,5\%) no estágio I, 6 (40\%) no estágio II e 7 (46,5\%) no estágio III. As médias de PA sistólica e diastólica e freqüência cardíaca (FC) aferidas no consultório

\begin{tabular}{|lcccc} 
& \multicolumn{2}{c}{ G1 $(\mathbf{n = 1 1})$} & \multicolumn{2}{c}{ G2 $(\mathbf{n = 1 5})$} \\
& 10 MAPA & 2 o MAPA & 10 MAPA & 2 ㅇ MAPA \\
\hline PASV & $146 \pm 14$ & $145 \pm 14$ & $150 \pm 14$ & $148 \pm 20$ \\
\hline PASS & $135 \pm 17$ & $135 \pm 19$ & $136 \pm 12$ & $137 \pm 19$ \\
\hline PADV & $91 \pm 11$ & $89 \pm 8$ & $92 \pm 7$ & $90 \pm 10$ \\
\hline PADS & $79 \pm 11$ & $80 \pm 12$ & $78 \pm 7$ & $79 \pm 9$ \\
\hline FCV & $80 \pm 7$ & $83 \pm 9$ & $79 \pm 12$ & $81 \pm 10$ \\
\hline FCS & $71 \pm 10$ & $73 \pm 10$ & $68 \pm 9$ & $69 \pm 10$ \\
\hline
\end{tabular}

* Não houve diferença estatisticamente significante em todas as medidas avaliadas pela MAPA entre os dois grupos e dentro de cada grupo quando comparada a primeira e a segunda MAPA. PASV $=P A$ sistólica na vigília; $P A S S=P A$ sistólica durante $o$ sono; $P A D V=P A$ diastólica na vigília; $P A D S=P A$ diastólica durante o sono; $F C V=F C$ na vigília; $F C S=F C$ durante o sono.

Tabela 1 - Medidas pressóricas avaliadas pela MAPA

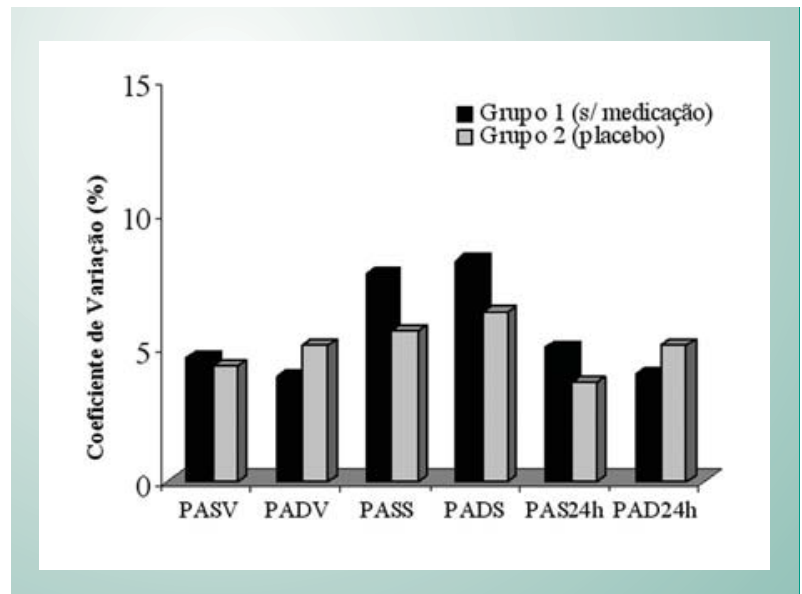

Fig. 1 - Coeficientes de variação das medidas pressóricas determinadas pela MAPA.

$P A S V=P A$ sistólica na vigília; $P A D V=P A$ diastólica na vigília; $P A S S=P A$ sistólica durante o sono; $P A D S=P A$ diastólica durante $o$ sono; $P A S 24 h=P A$ sistólica nas 24h; PAD24h=PA diastólica nas $24 h$. foram $173 \pm 20,98 \pm 7$ e $73 \pm 11$, respectivamente para o G1, e 166 $\pm 20,97 \pm 9$ e $77 \pm 9$, respectivamente para o G2.

Ao avaliarmos o CV da PA sistólica na vigília (PASV), PA diastólica na vigília (PADV), PA sistólica nas 24 horas (PAS24h) e PA diastólica nas 24 horas (PAD24h), encontramos valores de $4,6 \%, 3,9 \%, 5,0 \%$ e $4,0 \%$ no G1, e $4,3 \%, 5,1 \%, 3,7 \%$ e $5,1 \%$ no G2, respectivamente (fig. 1). Foi também determinado o CV da PA sistólica e diastólica durante o sono (PASS e PADS) para G1 (7,7\% e 8,2\%) e para G2 (5,6\% e $6,3 \%)$. O CV da freqüência cardíaca na vigília e durante o sono foi: $\mathrm{G} 1=5,9 \%$ e 9,0\%; G2 $=6,9 \%$ e 5,8\%, respectivamente. Analisando o total de pacientes $(\mathrm{N}=26)$, todas as variáveis mostraram fortes correlações entre a primeira e a segunda MAPA (PASV, $r=0,76 ; \mathrm{P}<0,001 ; \mathrm{PADV}, \mathrm{r}=0,65 ; \mathrm{p}<0,001$; PA sistólica nas 24h, $r=0,77 ; p<0,001 ; P A$ diastólica nas 24h, $r=0,70 ; p<0,001 ;$ PASS, $r=0,62 ; p<0,001 ;$ PADS, $r=0,52$; $p<0,01)$. Ocorreram também correlações entre a PA sistólica e a diastólica mensuradas no consultório e a PA sistólica e a diastólica nas 24 horas $(r=0,65 ; p<0,001 ; r=0,57 ; p<0,01)$, respectivamente. Quando comparamos os valores de DS sistólico e diastólico da primeira e da segunda MAPA, no G1 e G2 não encontramos diferença significante $(8,8 \%(-2,0$ a 14,3$)$ versus $5,6 \%(-1,7$ a 20,7$), \mathrm{NS}$; e $13,7 \%(5,5$ a 18,7$)$ versus $9,0 \%(-1$ a 30,9$)$, NS no G1. e $8,9 \%(-1,2$ a 17$)$ versus $6,1 \%(0$ a 19,7$), \mathrm{NS}$; e $14,4 \%(2,2$ a 31,1$)$ versus $13,2 \%(4,5$ a 19,4$)$, NS no G2, respectivamente). Finalmente, analisando em conjunto os dois grupos, quando consideramos como $10 \%$ o valor de corte para o descenso noturno para separar os "dippers" dos "non-dippers", encontramos que $42 \%$ dos diabéticos hipertensos mudaram de categoria no DS sistólico e 46 \% no DS diastólico após a repetição da monitorização, em um intervalo de 1 a 10 meses. No G1, mudaram de categoria 3 de 11 pacientes (27\%) no DS sistólico, e 6 de 11 pacientes (54\%) no DS diastólico; e no G2 isso ocorreu em 8 de 15 pacientes (53\%) para o DS sistólico, e em 6 de 15 pacientes (40\%) no DS diastólico. Quando comparamos dentro de cada grupo o porcentual de pacientes que mudaram de categoria não encontramos diferença significante.

\section{Discussão}

Os resultados do nosso estudo mostraram que os valores pressóricos médios avaliados pela monitorização ambulatorial da pressão arterial apresentaram uma boa reprodutibilidade e não foram afetados pelo efeito placebo. Esses achados são similares a poucos dados na literatura ${ }^{13,22}$.

Já descrevemos previamente (dados não publicados) que valores de PA sistólica durante o sono em diabéticos hipertensos foram relacionados com uma alta prevalência de hipertensão do ventrículo esquerdo; todavia, quando usado o descenso noturno da PA, esse efeito não foi observado. Isso provavelmente ocorreu em decorrência da baixa reprodutibilidade do descenso da PA durante o sono nesses pacientes. Reforçando essa hipótese, o presente estudo relatou que $42 \%$ dos pacientes hipertensos com DM mudaram de categoria (dipping/non-dipping) em uma segunda medida realizada após um intervalo de 1 a 10 meses. Verdecchia e cols. ${ }^{23}$ documentaram que $73 \%$ dos pacientes hipertensos permaneceram na mesma categoria (dipping/non-dipping) 


\section{Artigo Original}

depois de realizada uma segunda MAPA após um intervalo de três a cinco dias, enquanto $27 \%$ mudaram de um grupo para outro. Nos dados de Stenehjem e Os ${ }^{24}$, 82\% dos pacientes tiveram o seu padrão noturno (non-dipping) convertido para dipping depois de medidas repetidas. Essa discreta melhor reprodutibilidade do descenso noturno em pacientes hipertensos comparados com os nossos pacientes com DM e hipertensão poderia ser justificada, em parte, por outros fatores que influenciam o descenso noturno em diabéticos, entre eles o pobre controle glicêmico ${ }^{25}$ e a neuropatia autonômica diabética ${ }^{26,27}$.

Em relação às variações circadianas da PA em pacientes com diabetes tipo 1, variabilidade anormal persistente parece ocorrer precocemente e com freqüência naqueles com aumento da excreção urinária de albumina. A perda do descenso noturno (non-dippers) avaliada em duas MAPA foi observada em $80 \%$, 58\%, 18\% e 10\% de pacientes diabéticos com proteinúria, microalbuminúria, normoalbuminúria e grupo controle, respectivamente ${ }^{28}$, mostrando que a freqüência dessa anormalidade aumenta com a progressão de nefropatia incipiente $e^{28,29,30}$. Todavia, no presente estudo, todos os pacientes apresentaram níveis normais de creatinina e proteinúria nas 24 horas. Portanto, a nefropatia diabética não poderia justificar a elevada variabilidade do descenso noturno da PA em nosso grupo.

Dados da literatura avaliando a reprodutibilidade com medidas seriadas de MAPA, seguidas de MAPA realizadas após a retirada das medicações anti-hipertensivas e com uso de placebo em hipertensos com diabetes tipo 2 não são de nosso conhecimento. Asmar e cols. ${ }^{31}$ observaram em pacientes com hipertensão que a média da PA de 24 horas medida pela MAPA não foi afetada pelo placebo. Em pacientes com hipertensão leve ou moderada, Zakopoulos e cols. ${ }^{32}$ demonstraram que a PA sistólica e diastólica medida a

\section{Referências}

1. Feher MD. Hypertension in non-insulin dependent diabetes mellitus and its management. Postgrad Med J. 1991; 67: 938-46.

2. Nemcova H. Ambulatory monitoring of blood pressure in the treatment of hypertension in diabetics. Vnitr Lek. 2003; 49(12): 938-42.

3. Sowers JR, Khoury S. Diabetes and hypertension: a review. Prim Care. 1991; 18: 509-24.

4. Knowler WC, Bennet PH, Ballintine EJ. Increased incidence of retinopathy in diabetes with elevated blood pressure: a six-year follow-up study in Pima Indians. N Engl J Med. 1980; 302: 645-50.

5. The National High Blood Pressure Education Program Working Group. National high blood pressure education program working group on hypertension in diabetes. Hypertension. 1994; 23: 145-58.

6. Waeber B, Rutschmann B, Nüssberger J, Brunner HR. Evaluation of antihypertensive therapy: discrepancies between office and ambulatory recorded blood pressure. J Hypertens. 1991; 9(Suppl 3): S53-6.

7. Lurbe E, Redon J. Reproducibility and validity of ambulatory blood pressure monitoring in children. Am J Hypertens. 2002; 15: S69-73.

8. Mancia G, Parati G. Ambulatory blood pressure monitoring and organ damage. Am Heart J. 2000; 36: 894-900. cada hora, a freqüência cardíaca e a queda da PA durante o sono foram reprodutíveis em quatro MAPA realizadas em um período de quatro meses. Adicionalmente, Korner e cols. ${ }^{33}$, estudando crianças com diabetes tipo 1, concluíram que os valores de PA avaliados pela MAPA foram reprodutíveis apesar de marcadores de características individuais. Entretanto, Davies e cols. ${ }^{34}$ demonstraram que o mesmo equipamento de MAPA causou grande perturbação do sono, modificando a PA que estavam tentando mensurar. Outras causas para a variabilidade de PA nas 24 horas incluem problemas metodológicos com a MAPA durante a noite em razão de uma variabilidade hidrostática diferente entre o cuff do aparelho e o coração, nível de atividade durante o dia e diminuição da acurácia dos monitores ao mensurar níveis muito elevados de PA ${ }^{17,18,35}$. Davies e cols. ${ }^{34}$ superestimaram a PA sistólica em 10 mmHg (ABP, Oxford Medical) e em 6 mmHg (TM 2420, A\&D), enquanto a PA diastólica não foi afetada. Esse distúrbio poderia subestimar a queda pressórica durante o sono. Entretanto, outros monitores (SpaceLabs e Sandoz Pressure System) não mostraram esse mesmo problema em um estudo com avaliação concomitante da pressão intra-arterial ${ }^{36}$.

Por conseguinte, nosso estudo sugere que a MAPA (Spacelabs-90207, Inc. Redmond, WA - USA) é um instrumento confiável para monitorar as variações de PA e confirma sua confiabilidade para uso em pacientes hipertensos com diabete melito tipo 2 .

Financiamento: Conselho Nacional de Desenvolvimento Científico e Tecnológico - CNPq.

\section{Potencial Conflito de Interesses}

Declaro não haver conflitos de interesses pertinentes.
9. Pickering TG, Obrien E. Second International Consensus Meeting on 24hours ambulatory blood pressure measurement: consensus and conclusions. J Hypertens. 1991; 9 (Suppl 8): S2-6.

10. Primeiro Consenso Brasileiro para o uso da monitorização ambulatorial da pressão arterial nas 24 horas. Arq Bras Cardiol. 1993; 60 (2): 129-34.

11. Fotherby MD, Potter JF. Reproducibility of ambulatory and clinic blood pressure measurements in elderly hypertensive subjects. J Hypertens. 1993; 11: 573-9.

12. Mansoor GA, McCabe EJ, White WB. Long-term reproducibility of ambulatory blood pressure. J Hypertens. 1994; 12: 703-8.

13. Nielsen F, Rossing P, Bang L, Svendsen TL, Gall MA, Smidt UM. On the mechanisms of blunted nocturnal decline in arterial blood pressure in NIDDM patients with diabetic nephropathy. Diabetes 1995; 44: 783-9.

14. Bezdah L, Ouerdiane D, Kamoun M, Abid N, Lamine F, Kasri R. Value of ambulatory blood pressure monitoring in blood pressure profile determination in type 2 diabetes. Tunis Med. 2002; 80 (12): 769-74.

15. Manning G, Rushton L, Donnelly R, Millar-Craig MW. Variability of diurnal changes in ambulatory blood pressure and nocturnal dipping status in untreated hypertensive and normotensive subjects. Am J Hypertens. 2000; 13 (9):1035-8. 
16. Cuspidi C, Meani S, Salerno M, Valerio C, Fusi V, Severgnini B. Reproducibility of nocturnal blood pressure fall in early phases of untreated essential hypertension: a prospective observational study. J Hum Hypertens. 2004; 18(7): 503-9

17. Palatini P. Reliability of ambulatory blood pressure monitoring. Blood Press Monit. 2001; 6 (6): 291-5.

18. Parati G. Blood pressure reduction at night: sleep and beyond. J Hypertens 2000; 18 (12): 1725-9.

19. The fifth report of the Joint National Committee on Detection. Evaluation, and Treatment of High Blood Pressure (JNC V). Arch Intern Med. 1993; 153 (2): 154-83.

20. IV Brazilian Guideline in Arterial Hypertension Work Groups. Arq Bras Cardiol. 2004; 82 (Suppl 4): 7-22.

21. National Diabetes Data Group. Classification and diagnosis of diabetes mellitus and other categories of glucose intolerance. Diabetes. 1979; 28: 1039-57.

22. Prisant LM, Bottini P, Carr AA. Ambulatory blood pressure monitoring: methodologic issues. Am J Nephrol. 1996; 16(3): 190-201.

23. Verdecchia P, Schillaci G, Gatteschi C, Zampi I, Porcellati C. Quantitative assessment of day-to-day spontaneous variability in non-invasive ambulatory blood pressure measurements in essential hypertension. J Hypertens. 1991; 9 (Suppl 6): S322-3.

24. Stenehjem AE, Os I. Reproducibility of blood pressure variability, white-coat effect and dipping pattern in untreated, uncomplicated and newly diagnosed essential hypertension. Blood Press. 2004; 13(4): 214-24.

25. Hansen KW, Pedersen MM, Marshal SM, Christiansen JS, Mogensen CE. Circadian variation of blood pressure in patients with diabetic nephropathy. Diabetologia. 1992; 35: 1074-9.

26. Spallone V, Gambardella S, Maiello MR, Barini A, Frontoni S, Menzinger G. Relationship between autonomic neuropahty, 24-h blood pressure profile, and nephropathy in normotensive IDDM patients. Diabetes Care. 1994; 17: 578-84.
27. Hornung RS, Mahler RF, Raftery EB. Ambulatory blood pressure and heart rate in diabetic patients: an assessment of autonomic function. Diabetic Med. 1989; 6: 579-85.

28. Lurbe E, Redon J, Pascual JM, Tacons J, Alvarez V. The spectrum of circadian blood pressure changes in type I diabetic patients. J Hypertens. 2001; 19 (8): 1421-8.

29. Hansen KW, Poulsen PL, Christiansen JS, Mogensen CE. Determinants of 24-h blood pressure in IDDM patients. Diabetes Care. $1995 ; 18(4)$ : 529-35.

30. Cohen CN, Albanesi FM Fo, Gonçalves MFR, Gomes MB. Descenso pressórico durante o sono e microalbuminúria em pacientes normotensos com diabetes tipo 1. Endocrinol Metabol. 2001; 45 (3): 271-7.

31. Asmar R, Boutelant S, Chaignon M, Guedon J, Topouchian J, Mallion JM Repeated measurements of non-invasive ambulatory blood pressure: distinction between reproducibility and the proper effect of placebo. Blood Press Monit. 1996; 1 (3): 283-8.

32. Zakopoulos NA, Nanas SN, Lekakis JP, Vemmos KN, Kotsis VT, Pitiriga VC. Reproducibility of ambulatory blood pressure measurements in essential hypertension. Blood Press Monit. 2001; 6 (1): 41-5.

33. Korner A, Pataki V, Dobos M, Madacsy L, Miltenyi M, Tulassay T. Reproducibility of erythrocyte sodium-lithium countertransport activity and ambulatory blood pressure measurements in type 1 diabetes mellitus. Acta Diabetol. 1998; 35 (2): 104-8.

34. Davies RJO, Jenkins NE, Stradling JR. Effect of measuring ambulatory blood pressure on sleep and on blood pressure during sleep. BMJ. 1994; 308: 820-3.

35. Steen MS, Lenders JW, Graafsma SJ, den Arend J, Thien T. Reproducibility of ambulatory blood pressure monitoring in daily practice. J Hum Hypertens. 1999; 13 (5): 303-8.

36. Villani A, Parati G, Groppelli A, Omboni S, di Rienzo M, Mancia G. Noninvasive automatic blood pressure monitoring does not attenuate nighttime hypotension: evidence from $24 \mathrm{~h}$ intraarterial blood pressure monitoring. Am J Hypertens. 1992; 5: 744-7. 